\title{
An Optimal Explicit Guidance Algorithm for Terminal Descent Phase of Lunar Soft Landing
}

\author{
Avijit Banerjee* and Radhakant Padhi ${ }^{\dagger}$ \\ Indian Institute of Science, Bangalore, Karnataka, 560012, India
}

\begin{abstract}
An explicit guidance algorithm for multi-constrained terminal descent phase of lunar soft landing is presented in this paper. A minimum jerk guidance is designed and extended for this purpose to achieve the terminal control and state constraints. The closed form jerk expression, obtained using the minimum jerk guidance is analyzed to obtain an explicit expression for acceleration command, which is the physical control variable for the guidance loop. The guidance formulation ensures the minimum rate of change of acceleration and vertical touchdown of the spacecraft towards a designated landing site with high terminal accuracy. The design features of the proposed guidance law are demonstrated using simulation results.
\end{abstract}

\section{Nomenclature}

$v_{x}, v_{z}$

$a_{x}, a_{z}$

$u_{x}, u_{z}$

$J$

$\lambda$

$\lambda_{x}, \lambda_{z}, \lambda_{v_{x}}, \lambda_{v_{z}}, \lambda_{a_{x}}, \lambda_{a_{z}}$

$\lambda_{x f}, \lambda_{z f}, \lambda_{v_{x f}}, \lambda_{v_{z f}}, \lambda_{a_{x f}}, \lambda_{a_{z f}}$

$t$

$t_{f}$

$t_{g o}$

$t_{g o_{0}}$

$Z_{x}, Z_{z}$

$V_{x}, V_{z}$

$A_{x}$

$c_{1 x}, c_{2 x}, c_{3 x}, c_{1 z}, c_{2 z}, c_{3 z}$

$J_{a}$

$J_{a x}$

$J_{a z}$

$\beta$

$x_{0}, z_{0}$

$v_{x 0}, v_{z 0}$

$a_{x 0}, a_{z 0}$

$x_{f}, z_{f}$

$v_{x f}, v_{z f}$

$a_{x f}, a_{z f}$

$g$
Downrange, $\mathrm{m}$

Altitude, $m$

Horizontal and vertical components of velocity, $\mathrm{m} / \mathrm{s}$

Horizontal and vertical components of acceleration, $\mathrm{m} / \mathrm{s}^{2}$

Horizontal and vertical components of jerk, m/ $\mathrm{s}^{3}$

Cost function for jerk minimization, $\mathrm{m}^{2} / \mathrm{s}^{5}$

Co-state vector

Components of co-state vector

Terminal components of co-state vector

Time, s

Final flight time, $\mathrm{s}$

Available flight time, $\mathrm{s}$

Initial available flight time, s

Zero effort miss along downrange and altitude, $\mathrm{m}$

Zero effort velocity miss along downrange and altitude, $\mathrm{m} / \mathrm{s}$

Zero effort acceleration miss along downrange, $\mathrm{m} / \mathrm{s}^{2}$

Integration Constants

Cost function for acceleration minimization, $\mathrm{m}^{2} / \mathrm{s}^{3}$

Cost function for acceleration minimization along downrange, $\mathrm{m}^{2} / \mathrm{s}^{3}$

Cost function for acceleration minimization along altitude, $\mathrm{m}^{2} / \mathrm{s}^{3}$

Look angle, Deg

Initial position components, $\mathrm{m}$

Initial velocity components, $\mathrm{m} / \mathrm{s}$

Initial acceleration components, $\mathrm{m} / \mathrm{s}^{2}$

Terminal position components, $\mathrm{m}$

Terminal velocity components, $\mathrm{m} / \mathrm{s}$

Terminal acceleration components, $\mathrm{m} / \mathrm{s}^{2}$

gravitational acceleration, , $\mathrm{m} / \mathrm{s}^{2}$

*PhD Student, Department of Aerospace Engineering, Indian Institute of Science, Bangalore, India.

${ }^{\dagger}$ Professor, Dept. of Aerospace Engineering, IISc, Bangalore, India and Associate Fellow, AIAA 


$\begin{array}{ll}H & \text { Hamiltonian } \\ X \in \mathbb{R}^{6 \times 1} & \text { State Vector } \\ P \in \mathbb{R}^{6 \times 6} & \text { System matrices } \\ Q \in \mathbb{R}^{6 \times 1} & \text { Input matrices }\end{array}$

\section{Introduction}

Over the years, space exploration has become a potential platform for demonstrating technological prowess of a nation. One key objective of space exploration is precise and safe landing of a space vehicle over the unknown territory of a celestial body. An autonomous descent guidance for landing on moon, which has to be essentially a soft landing, starts from the perilune, the nearest point of the elliptical orbit (about $18 \mathrm{~km}$ ) from the lunar surface. The descent trajectory typically involves three intermediate phases, viz., rough braking, fine braking and terminal descent phase. The phase-I (rough braking) incorporates a reverse thrust braking mechanism to decelerate the spacecraft from initial high orbital velocity to relatively a lower velocity. The objective of the fine braking phase is to guide the spacecraft towards a designated landing site with a residual altitude $(100 \mathrm{~m})$. At the end of the braking phase, the spacecraft is placed just above the predetermined landing site with near zero velocity. At this point, a simple vertical terminal descent may not be adequate as the presence of process noise during fine braking may cause a significant deviation of the downrange which leads to deviation of the spacecraft from desired location. Following the entire successful journey from earth, the possibility of landing over a small boulder or large pebble may tumble down the spacecraft. Hence a vision based autonomous re-targeting is necessary. Onboard image processing unit captures the local image of lunar surface and provide a safe landing site. The objective of the autonomous re-targeting guidance is to drive the spacecraft towards the selected landing site with zero velocity. Also, the terminal orientation of the spacecraft needs to be vertical with respect to the local lunar surface.

Many researches have been carried out to formulate a terminal guidance with autonomous re-targeting feature. A path shaping guidance ${ }^{1}$ for terminal soft landing is presented by Colin et al. The guidance formulation provides an analytical solution based on gravity turn approach. ${ }^{2}$ Adopting the nonlinear backstepping control approach Feng et al. ${ }^{3}$ addressed the terminal descent problem in Intergrated Guidance and Control (IGC) framework. A hybrid thrust-tether based terminal guidance strategy in presented in. ${ }^{4}$ D'souza $^{5}$ framed a fuel optimal feedback guidance law based on acceleration minimization approach for planetary landing. The resulting guidance law is commonly known as constrained terminal velocity guidance (CTVG). The inherent similarities of the CTVG with the generalized explicit guidance (GENEX) are demonstrated by Guo et al. ${ }^{6}$ Although the close form acceleration expression obtained from CTVG is applicable for onboard implementation, it is inadequate to ensure the terminal vertical orientation of the spacecraft and also the trajectory generated using CTVG may results into subsurface travel. Also, Intercept Angle Control Guidance (IACG), as a combination of CTVG and Free Terminal Velocity Guidance (FTVG), is proposed to address the look-angle constraints. However, the IACG may not satisfie the soft landing requirement as the formulation inherently poses a directionally constrained terminal velocity vector with velocity magnitude being free parameter. To ensure the terminal orientation of lunar module, the desired terminal acceleration is incorporated with the cost function of CTVG as a soft constraint. ${ }^{7}$ Depending on the choice of the sensitivity parameter of the additional soft constraint, the formulation incorporates tradeoff between the fuel optimality and terminal control constraint. The augmentation of the additional soft constraint ${ }^{7}$ eventually leads to a modified expression of the final time, derived based on transversality conditions. An improved CTVG, ${ }^{8}$ prevents the spacecraft trajectory from sub surface travel. But the modified acceleration command may results in an unacceptable terminal position and velocity error.

Zhang et al. ${ }^{8}$ introduced the concept of pseudo control as time derivative of acceleration to attain the terminal state and control constraints simultaneously . In order to eliminate the unacceptable terminal error, the Model predictive Static Programming (MPSP) is proposed for generating the guidance command. The MPSP based algorithm provides an onboard computable fast numerical guidance with high precision terminal accuracy. A similar concept like pseudo control as the time derivative of acceleration has been adopted by Uchiyama et al. ${ }^{9}$ A unified feedback guidance law, based on jerk minimization, in constrast to the earlier acceleration minimization approach is introduced. The closed form jerk expression obtained using calculus 
of variation, ${ }^{9}$ addresses all the terminal constraints (state and control) for the retargeting phase of the lunar landing. The final flight time is computed based on the transversality conditions and results into a sixth order polynomial of available flight time. A minimum jerk based missile guidance law was given by Grinfeld et al. ${ }^{10}$ Here a different solution approach is considered based on state transition matrix. Although the closed form solution of the optimal jerk is presented, the jerk commands need to be integrated numerically to obtain equivalent accelerations as physical guidance command, in order to implement the same in actual guidance-loop. The numerical integration may involve sensors biases and is prone to integration errors.

In this paper, the minimum jerk guidance has been designed for the re-targeting phase of the lunar soft

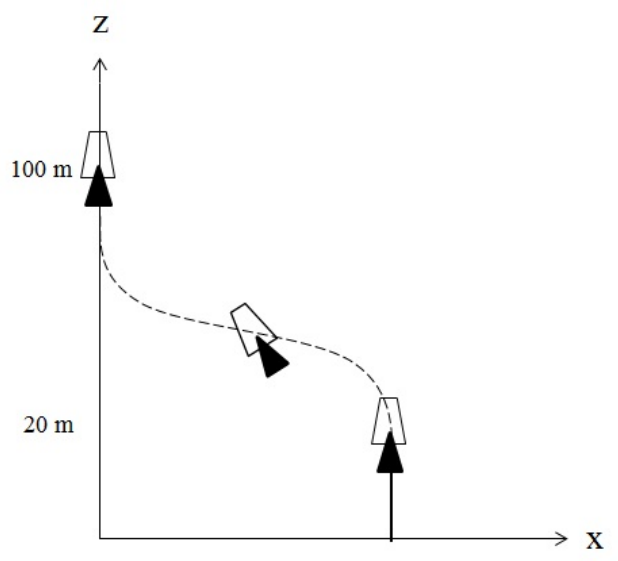

Figure 1. Schematic spacecraft trajectory and reference frame

landing. Based on the state feedback jerk expression, a closed form solution of the acceleration command has been derived. That eliminates the requirement of numerical integration. The minimum jerk based guidance command provides a family of acceleration profiles based on initial choice of available flight time $\left(t_{g o_{0}}\right)$, such that all of them ensure the desired terminal constraints (position, velocity and look angle) for the soft lunar landing with high terminal accuracy. Along with aforementioned characteristics, the minimum jerk based guidance formulation assures minimum rate of change of acceleration which is much desirable from propulsion perspective as the thrust engine has a physical limitation to respond to the change in acceleration demand.

\section{Problem Objective}

At the end of the braking phase, the lunar module is supposed to be placed at an altitude of $100 \mathrm{~m}$ from the local lunar surface with near zero velocity and vertical orientation. The velocity and vertical look angle requirement is necessary to capture good quality local terrain images (free from motion blur). The image processing unit determines a safe landing site based on the local terrain images captured by onboard camera. The autonomous re-targeting guidance objective is to drive the spacecraft safely towards the determined landing site and ensure the terminal velocity and vertical orientation requirements.

The lunar terminal descent initiate at about an altitude of $100 \mathrm{~m}$, where the lunar surface can be reasonably considered as flat and gravitational acceleration is fairly constant. spacecraft dynamics in local inertial reference frame is the same adopted by D'souza ${ }^{5}$ which is described by the following equation of motion Eq.(1) The origin of the local reference frame is considered to be located on the local terrain, vertically below the position of the spacecraft at the end of braking phase $(100 \mathrm{~m}$ altitude as shown in Fig.(1)). The origin and the initial position of spacecraft together determine the ' $\mathrm{z}$ ' axis. The vector, from the origin towards the re-targeted landing site construct the ' $x$ ' axis. The re-targeting maneuver of the spacecraft is considered to be confined in planar motion (' $\mathrm{x}-\mathrm{z}$ ' plane) as shown in Fig.(1). where, $(x, z)$ represents the position of the spacecraft described in local inertial frame of reference, $\left(v_{x}, v_{z}\right)$ and $\left(a_{x}, a_{z}\right)$ describe the velocity and acceleration components respectively of the spacecraft motion in respective directions. The lunar gravitational acceleration is denoted by $g$. The time derivative of acceleration components $\left(u_{x}, u_{y}\right)$ 
essentially represent the jerks, in respective directions, which are considered to be the control variables. The spacecraft initial position $\left(x_{0}, z_{0}\right)$, velocity $\left(v_{x_{0}}, v_{z_{0}}\right)$, and acceleration $\left(a_{x_{0}}, a_{z_{0}}\right)$ of the re-targeting phase are essentially obtained from the terminal states of the braking phase. Similarly the desired terminal states are given by, $\left(x_{f}, z_{f}\right),\left(v_{x f}, v_{z f}\right)$ and $\left(a_{x f}, a_{z f}\right)$ respectively.

$$
\begin{aligned}
& \dot{x}=v_{x} \\
& \dot{z}=v_{z} \\
& \dot{v}_{x}=a_{x} \\
& \dot{v}_{z}=a_{z}+g \\
& \dot{a}_{x}=u_{x} \\
& \dot{a}_{z}=u_{z}
\end{aligned}
$$

In order to accomplish a successful soft landing mission, very precise spacecraft motion must be carried out during the terminal descent phase. To satisfy all the terminal constraints accurately and also to reduces the possibility of subsurface travel, the terminal descent phase is divided into two intermediate sub phases. The phase-I (re-targeting phase) is designed to drive the spacecraft from $100 \mathrm{~m}$ altitude and place it just above the re-targeted landing site of about $20 \mathrm{~m}$ altitude. At the end of phase-I, only the horizontal velocity component $v_{x f}=0$ must be nullified. During the phase-II(vertical descent phase) the spacecraft is only allowed to perform an unidirectional motion and from $20 m$ it descends down vertically to $2 m$ altitude with zero velocity. The introduction of the additional sub-phase in guidance design provides an auxiliary safe guard (of $20 \mathrm{~m}$ ) to subsurface travel. Also, it reduces the possibility of the terminal residual errors. After reaching $2 m$ the propulsion system needs to shut down to prevent the contamination of lunar dust over the solar panel and spacecraft is allowed to free fall with negligible impact.

\section{Generic Formulation of Minimum Jerk Guidance}

Although the constrained terminal velocity guidance, ${ }^{5}$ derived based on acceleration minimization provides the closed form feedback expression for acceleration, the guidance law is inadequate to ensure the terminal control constrain (vertical look angle). As the thrust engine is attached with the lunar module (along the roll axis), direction of thrust vector essentially represents the spacecraft orientation. The thrust vector orientation (look angle) is expressed in terms of acceleration components as

$$
\beta=\tan ^{-1}\left(\frac{a_{z}}{a_{x}}\right)
$$

To ensure the the terminal vertical orientation $\left(\beta=90^{\circ}\right)$, terminal horizontal acceleration component needs to be nullified $\left(a_{x f}=0\right)$. The explicit guidance law based on jerk minimization ${ }^{9}$ is presented in this section for the shake of completeness. The derivation of minimum jerk guidance considers the acceleration as the state variable. Hence the formulation inherently poses the desired terminal look angle constraint. Moreover, the resulting guidance law comes with an additional freedom of choice for the initial acceleration. This additional freedom can be used to assure control continuity between the braking and the re-targeting phases. The control continuity essentially leads to a smooth spacecraft trajectory. The minimum jerk guidance is formulated as the following optimal control problem

$$
\begin{aligned}
\min _{\dot{a}_{x}, \dot{a}_{z}} J & =\frac{1}{2} \int_{0}^{t_{f}}\left(\dot{a}_{x}^{2}+\dot{a}_{z}^{2}\right) d t \\
& =\min _{u_{x}, u_{z}}\left(\frac{1}{2} \int_{0}^{t_{f}}\left(u_{x}^{2}+u_{z}^{2}\right) d t\right)
\end{aligned}
$$

The objective is to obtain a closed form jerk expression that minimizes the cost function of Eq.(3) subjected to the system dynamics Egn.(1) along with the initial conditions $x(0)=x_{0}, z(0)=z_{0}, v_{x}(0)=v_{x 0}, v_{z}(0)=$ $v_{z 0}, a_{x}(0)=a_{x 0}, a_{z}(0)=a_{z 0}$ and the following terminal conditions needs to be ensured. 
$x\left(t_{f}\right)=x_{f}, z\left(t_{f}\right)=z_{f}, v_{x}\left(t_{f}\right)=v_{x f}, v_{z}\left(t_{f}\right)=v_{z f}, a_{x}\left(t_{f}\right)=a_{x f}, a_{z}\left(t_{f}\right)=a_{z f}$. Considering the co-state vector

$$
\lambda^{T}=\left[\begin{array}{llllll}
\lambda_{x} & \lambda_{z} & \lambda_{v_{x}} & \lambda_{v_{z}} & \lambda_{a_{x}} & \lambda_{a_{z}}
\end{array}\right]
$$

The Hamiltonian for the above problem is formulated as,

$$
H=\frac{1}{2}\left(u_{x}^{2}+u_{z}^{2}\right)+\lambda_{x} v_{x}+\lambda_{z} v_{z}+\lambda_{v_{x}} a_{x}+\lambda_{v_{z}}\left(a_{z}+g\right)+\lambda_{a_{x}} u_{x}+\lambda_{a_{z}} u_{z}
$$

Using the calculus of variation the co-state dynamics is obtained as

$$
\begin{aligned}
& \dot{\lambda}_{x}=0 \\
& \dot{\lambda}_{z}=0 \\
& \dot{\lambda}_{v_{x}}=-\lambda_{x} \\
& \dot{\lambda}_{v_{z}}=-\lambda_{z} \\
& \dot{\lambda}_{a_{x}}=-\lambda_{v_{x}} \\
& \dot{\lambda}_{a_{z}}=-\lambda_{v_{z}}
\end{aligned}
$$

The optimal control equation in terms of co-state variables is presented as

$$
\begin{aligned}
& u_{x}+\lambda_{a_{x}}=0 \\
& u_{z}+\lambda_{a_{z}}=0
\end{aligned}
$$

considering the terminal co-state variables as follows,

$$
\begin{gathered}
\lambda_{x}\left(t_{f}\right)=\lambda_{x f} \\
\lambda_{z}\left(t_{f}\right)=\lambda_{z f} \\
\lambda_{v_{x}}\left(t_{f}\right)=\lambda_{v_{x f}} \\
\lambda_{v_{z}}\left(t_{f}\right)=\lambda_{v_{z f}} \\
\lambda_{a_{x}}\left(t_{f}\right)=\lambda_{a_{x f}} \\
\lambda_{a_{z}}\left(t_{f}\right)=\lambda_{a_{z f}}
\end{gathered}
$$

Denoting $t_{g o}=t_{f}-t$, the solution of the co-state equation is represented as

$$
\begin{aligned}
\lambda_{x} & =\lambda_{x f} \\
\lambda_{z} & =\lambda_{z f} \\
\lambda_{v_{x}} & =\lambda_{x f} t_{g o}+\lambda_{v_{x f}} \\
\lambda_{v_{z}} & =\lambda_{z f} t_{g o}+\lambda_{v_{z f}} \\
\lambda_{a_{x}} & =\frac{\lambda_{x f}}{2} t_{g o}^{2}+\lambda_{v_{x f}} t_{g o}+\lambda_{a_{x f}} \\
\lambda_{a_{z}} & =\frac{\lambda_{z f}}{2} t_{g o}^{2}+\lambda_{v_{z f}} t_{g o}+\lambda_{a_{z f}}
\end{aligned}
$$

Using the solution of the co-state equations, the optimal control equations are rewritten as

$$
\begin{aligned}
& u_{x}=-\left(\frac{\lambda_{x f}}{2} t_{g o}^{2}+\lambda_{v_{x f}} t_{g o}+\lambda_{a_{x f}}\right) \\
& u_{z}=-\left(\frac{\lambda_{z f}}{2} t_{g o}^{2}+\lambda_{v_{z f}} t_{g o}+\lambda_{a_{z f}}\right)
\end{aligned}
$$


Putting the optimal control expression into the system dynamics, the solution of the system states are obtained as

$$
\begin{aligned}
& x=\frac{\lambda_{x f}}{120} t_{g o}^{5}+\frac{\lambda_{v_{x f}}}{24} t_{g o}^{4}+\frac{\lambda_{a_{x f}}}{6} t_{g o}^{3}+\frac{a_{x f}}{2} t_{g o}^{2}-v_{x f} t_{g o}+x_{f} \\
& z=\frac{\lambda_{z f}}{120} t_{g o}^{5}+\frac{\lambda_{v_{z f}}}{24} t_{g o}^{4}+\frac{\lambda_{a_{z f}}}{6} t_{g o}^{3}+\frac{a_{x f}}{2} t_{g o}^{2}-v_{z f} t_{g o}+z_{f} \\
& v_{x}=-\frac{\lambda_{x f}}{24} t_{g o}^{4}-\frac{\lambda_{v_{x f}}}{6} t_{g o}^{3}-\frac{\lambda_{a_{x f}}}{2} t_{g o}^{2}-a_{x f} t_{g o}+v_{x f} \\
& v_{z}=-\frac{\lambda_{z f}}{24} t_{g o}^{4}-\frac{\lambda_{v_{z f}}}{6} t_{g o}^{3}-\frac{\lambda_{a_{z f}}}{2} t_{g o}^{2}-\left(a_{z f}+g\right) t_{g o}+v_{z f} \\
& a_{x}=\frac{\lambda_{x f}}{6} t_{g o}^{3}+\frac{\lambda_{v_{x f}}}{2} t_{g o}^{2}+\lambda_{a_{x f}} t_{g o}+a_{x f} \\
& a_{z}=\frac{\lambda_{z f}}{6} t_{g o}^{3}+\frac{\lambda_{v_{z f}}}{2} t_{g o}^{2}+\lambda_{a_{z f}} t_{g o}+a_{z f}
\end{aligned}
$$

Solving the above system of equations for $\lambda_{x f}, \lambda_{z f}, \lambda_{v_{x f}}, \lambda_{v_{z f}}, \lambda_{a_{x f}}$ and $\lambda_{a_{z f}}$ in terms of system state and putting that solution into the control expressions Eq.(7)and Eq.(8) the closed form solution for minimum jerk in respective directions are obtained.

$$
\begin{aligned}
& u_{x}=-\frac{60}{t_{g o}^{3}}\left(x-x_{f}+v_{x f} t_{g o}-\frac{a_{x f}}{2} t_{g o}^{2}\right)-\frac{36}{t_{g o}^{2}}\left(v_{x}-v_{x f}+a_{x f} t_{g o}\right)-\frac{9}{t_{g o}}\left(a_{x}-a_{x f}\right) \\
& u_{z}=-\frac{60}{t_{g o}^{3}}\left(z-z_{f}+v_{z f} t_{g o}-\frac{\left(a_{z f}+g\right)}{2} t_{g o}^{2}\right)-\frac{36}{t_{g o}^{2}}\left(v_{z}-v_{z f}+\left(a_{z f}+g\right) t_{g o}\right)-\frac{9}{t_{g o}}\left(a_{z}-a_{z f}\right)
\end{aligned}
$$

The closed form optimal jerk expressions obtained in Eq.(10) and Eq.(11) are capable to drive the spacecraft initial state to the desired terminal state and also ensures the terminal look angle constraint. The jerk expression obtained in Eq.(10) and Eq.(11) are more generic compared to Eq.(26a) and Eq. (26b), as obtained by Uchiyama ${ }^{9}$ and the desired terminal states $x_{f}, z_{f}, v_{x f}, v_{z f}$ and $a_{x f}$ are not necessarily to be zero.

\section{Analytic Expression of Acceleration as Physical Guidance Command}

Although the minimum jerk is obtained in state feedback form (Eq.(10) and Eq.(11), a typical guidance loop operates on acceleration command as the physical control variable. The jerk expression needs to be numerically integrated to obtain the acceleration demand. Hence, the minimum jerk guidance does not solely solves the purpose of an analytic guidance when it comes to the actual guidance application. At the beginning of formulation, jerk is introduced as a pseudo control variable as the time derivative of acceleration. After all the necessary mathematical derivations, it is desirable to obtain the physical control variable in explicit form. In this section an attempt has been made to obtain an explicit expression of acceleration command. As the expression of jerk involves all other system states, it demands the simultaneous solution of a coupled differential equations. Putting the jerk command into the system dynamics Eq.(1) and considering $\frac{d t_{g o}}{d t}=\frac{d}{d t}\left(t_{f}-t\right)=-1$ the following time varying state space equation is obtained.

$$
\frac{d X}{d t_{g o}}=P\left(t_{g o}\right) X+Q\left(t_{g o}\right)
$$

where, the state vector $X=\left[\begin{array}{llllll}x & z & v_{x} & v_{z} & a_{x} & a_{z}\end{array}\right]^{T}$ and the expressions for $P\left(t_{g o}\right)$ and $Q\left(t_{g o}\right)$ are represented as 


$$
\begin{aligned}
& P\left(t_{g o}\right)=\left[\begin{array}{cccccc}
0 & 0 & -1 & 0 & 0 & 0 \\
0 & 0 & 0 & -1 & 0 & 0 \\
0 & 0 & 0 & 0 & -1 & 0 \\
0 & 0 & 0 & 0 & 0 & -1 \\
\frac{60}{t_{g o}^{3}} & 0 & \frac{36}{t_{g o}^{2}} & 0 & \frac{9}{t_{g o}} & 0 \\
0 & \frac{60}{t_{g o}^{3}} & 0 & \frac{36}{t_{g o}^{2}} & 0 & \frac{9}{t_{g o}}
\end{array}\right] \\
& Q\left(t_{g o}\right)=\left[\begin{array}{c}
0 \\
0 \\
0 \\
0 \\
-\frac{60}{t_{g o}^{3}}\left(x_{f}-v_{x f} t_{g o}+\frac{a_{x f}}{2} t_{g o}^{2}\right)-\frac{36}{t_{g o}^{2}}\left(v_{x f}-a_{x f} t_{g o}\right)+\frac{9}{t_{g o}} a_{x f} \\
-\frac{60}{t_{g o}^{3}}\left(x_{f}-v_{x f} t_{g o}+\frac{a_{x f}}{2} t_{g o}^{2}\right)-\frac{36}{t_{g o}^{2}}\left(v_{x f}-a_{x f} t_{g o}\right)+\frac{9}{t_{g o}} a_{x f}
\end{array}\right]
\end{aligned}
$$

Solution of such time varying differential equations is not very trivial. To obtain the closed form acceleration expression an alternate approach has been adapted in the present work. The following terms are defined for brevity.

$$
\begin{aligned}
& Z_{x}=x-x_{f}+v_{x f} t_{g o}-\frac{a_{x f}}{2} t_{g o}^{2} \\
& V_{x}=v_{x}-v_{x f}+a_{x f} t_{g o} \\
& A_{x}=a_{x}-a_{x f} \\
& Z_{z}=z-z_{f}+v_{z f} t_{g o}-\frac{\left(a_{z f}+g\right)}{2} t_{g o}^{2} \\
& V_{z}=v_{z}-v_{z f}+\left(a_{z f}+g\right) t_{g o} \\
& A_{z}=a_{z}-a_{z f}
\end{aligned}
$$

According to GENEX guidance, ${ }^{11} Z_{x}, Z_{z}$ are termed as Zero Effort Miss (ZEM), $V_{x}, V_{z}$ are known as Zero Effort Velocity Miss (ZEVM) and $A_{x}, A_{z}$ can be called as Zero Effort Acceleration Miss (ZEAM). Combining Eq. (10) and Eq.(11), along with Eq.(13) and Eq.(14), the following jerk expressions are obtained.

$$
\begin{aligned}
& u_{x}=-\frac{60}{t_{g o}^{3}} Z_{x}-\frac{36}{t_{g o}^{2}} V_{x}-\frac{9}{t_{g o}} A_{x} \\
& u_{z}=-\frac{60}{t_{g o}^{3}} Z_{z}-\frac{36}{t_{g o}^{2}} V_{z}-\frac{9}{t_{g o}} A_{z}
\end{aligned}
$$

As $t_{g o}=t_{f}-t, \frac{d t_{g o}}{d t}=-1$, Differentiating Eq.(13) with respect to $t_{g o}$ and using Eq.(1) the following relations are obtained for ' $\mathrm{x}$ ' component

$$
\begin{aligned}
& \frac{d Z_{x}}{d t_{g o}}=-V_{x} \\
& \frac{d^{2} Z_{x}}{d t_{g o}^{2}}=-\frac{d V_{x}}{d t_{g o}}=A_{x} \\
& \frac{d^{3} Z_{x}}{d t_{g o}^{3}}=\frac{d A_{x}}{d t_{g o}}=-u_{x}
\end{aligned}
$$


A similar set of operation for ' $z$ ' component, using Eq.(14) and Eq.(1) leads to the following expressions

$$
\begin{aligned}
& \frac{d Z_{z}}{d t_{g o}}=-V_{z} \\
& \frac{d^{2} Z_{z}}{d t_{g o}^{2}}=-\frac{d V_{z}}{d t_{g o}}=A_{z} \\
& \frac{d^{3} Z_{z}}{d t_{g o}^{3}}=\frac{d A_{z}}{d t_{g o}}=-u_{z}
\end{aligned}
$$

Putting the expressions of Eq.(17) and Eq.(18) into Eq.(15) and Eq.(16), results a third order ordinary differential equation of Cauchy's form as shown below,

$$
\begin{aligned}
& t_{g o}^{3} \frac{d^{3} Z_{x}}{d t_{g o}^{3}}-9 \frac{d^{2} Z_{x}}{d t_{g o}^{2}} t_{g o}^{2}+36 \frac{d Z_{x}}{d t_{g o}}-60 Z_{x}=0 \\
& t_{g o}^{3} \frac{d^{3} Z_{z}}{d t_{g o}^{3}}-9 \frac{d^{2} Z_{z}}{d t_{g o}^{2}} t_{g o}^{2}+36 \frac{d Z_{z}}{d t_{g o}}-60 Z_{z}=0
\end{aligned}
$$

Substitution of $Z_{x}=t_{g o}^{m}$ in the Eq.(19) leads to the following equation of $m$

$$
m^{3}-12 m^{2}+47 m-60=0
$$

Solving the above of Eq.(21) values of $m$ are obtained as, $m=\{3,4,5\}$. Hence, $Z_{x}$ can be expressed as

$$
Z_{x}\left(t_{g o}\right)=c_{1 x} t_{g o}^{3}+c_{2 x} t_{g o}^{4}+c_{3 x} t_{g o}^{5}
$$

Similarly the expression for $Z_{z}\left(t_{g o}\right)$ can be obtained by solving Eq.(20) and the solution is given as

$$
Z_{z}\left(t_{g o}\right)=c_{1 z} t_{g o}^{3}+c_{2 z} t_{g o}^{4}+c_{3 z} t_{g o}^{5}
$$

where $c_{1 x}, c_{2 x}, c_{3 x}$ represent constants of integration and needs to be evaluated. Taking two successive derivatives of $Z_{x}\left(t_{g o}\right)$ and $Z_{z}\left(t_{g o}\right)$ with respect to $t_{g o}$ and using Eq.(17) following sets of algebraic relations are obtained.

$$
\begin{aligned}
& V_{x}\left(t_{g o}\right)=-\left(3 c_{1 x} t_{g o}^{2}+4 c_{2 x} t_{g o}^{3}+5 c_{3 x} t_{g o}^{4}\right) \\
& V_{z}\left(t_{g o}\right)=-\left(3 c_{1 z} t_{g o}^{2}+4 c_{2 z} t_{g o}^{3}+5 c_{3 z} t_{g o}^{4}\right) \\
& A_{x}\left(t_{g o}\right)=6 c_{1 x} t_{g o}+12 c_{2 x} t_{g o}^{2}+20 c_{3 x} t_{g o}^{3} \\
& A_{z}\left(t_{g o}\right)=6 c_{1 z} t_{g o}+12 c_{2 z} t_{g o}^{2}+20 c_{3 z} t_{g o}^{3}
\end{aligned}
$$

Substituting the $A_{x}$ and $A_{z}$ expression from Eq.(13) and Eq.(14) into Eq.(24) and Eq.(25), the explicit expression of acceleration command is presented as

$$
\begin{aligned}
& a_{x}=20 c_{3 x} t_{\text {go }}^{3}+12 c_{2 x} t_{g o}^{2}+6 c_{1 x} t_{g o}+a_{x f} \\
& a_{z}=20 c_{3 z} t_{g o}^{3}+12 c_{2 z} t_{g o}^{2}+6 c_{1 z} t_{g o}+a_{z f}
\end{aligned}
$$

As $t_{g o}=t_{f}-t$ hence at $t=\left.t_{f} t_{g o}\right|_{t=t_{f}}=0$, from the above expressions Eq.(26) and Eq.(27) one can verify that, the guidance formulation ensures the desired terminal acceleration and hence the desired look angle constraint is achieved. The explicit expression of the acceleration commands obtained in Eq.(26) and Eq.(27) are essentially polynomials of $t_{g o}$ with constant coefficients. These coefficients, appear as constants of integration, are computed in this subsection. Combining the zero effort terms (ZEM, ZEVM and ZEAM) 
for the $x$-components and evaluating those at initial time $\left(\left.t_{g o}\right|_{t=0}=t_{g o_{0}}\right)$, following set of algebraic equations are obtained.

$$
\left[\begin{array}{c}
Z_{x}\left(t_{g o_{0}}\right) \\
V_{x}\left(t_{g o_{0}}\right) \\
A_{x}\left(t_{g o_{0}}\right)
\end{array}\right]=\left[\begin{array}{ccc}
t_{g o_{0}}^{3} & t_{g o_{0}}^{4} & t_{g o_{0}}^{5} \\
-3 t_{g o_{0}}^{2} & -4 t_{g o_{0}}^{3} & -5 t_{g o_{0}}^{4} \\
6 t_{g o_{0}} & 12 t_{g o_{0}}^{2} & 20 t_{g o_{0}}^{3}
\end{array}\right]\left[\begin{array}{l}
c_{1 x} \\
c_{2 x} \\
c_{3 x}
\end{array}\right]
$$

where, $Z_{x}\left(t_{g o_{0}}\right), V_{x}\left(t_{g o_{0}}\right)$ and $A_{x}\left(t_{g o_{0}}\right)$ are obtained by putting $\left.t_{g o}\right|_{t=0}=t_{g o_{0}}$ into Eq.(13) as

$$
\begin{aligned}
& Z_{x}\left(t_{g o_{0}}\right)=x_{0}-x_{f}+v_{x f} t_{g o_{0}}-\frac{a_{x f}}{2} t_{g o_{0}}^{2} \\
& V_{x}\left(t_{g o_{0}}\right)=v_{x 0}-v_{x f}+a_{x f} t_{g o_{0}} \\
& A_{x}\left(t_{g o_{0}}\right)=a_{x 0}-a_{x f}
\end{aligned}
$$

Putting Eq.(29) into the solution of Eq.(28) the integration constants $\left(c_{1 x}, c_{2 x}\right.$ and $\left.c_{3 x}\right)$ are obtained as

$$
\begin{aligned}
& c_{1 x}=\frac{10}{t_{g o_{0}}^{3}}\left(x_{0}-x_{f}+v_{x f} t_{g o_{0}}-\frac{a_{x f}}{2} t_{g o_{0}}^{2}\right)+\frac{4}{t_{g o_{0}}^{2}}\left(v_{x 0}-v_{x f}+a_{x f} t_{g o_{0}}\right)+\frac{1}{2 t_{g o_{0}}}\left(a_{x 0}-a_{x f}\right) \\
& c_{2 x}=-\frac{15}{t_{g o_{0}}^{4}}\left(x_{0}-x_{f}+v_{x f} t_{g o_{0}}-\frac{a_{x f}}{2} t_{g o_{0}}^{2}\right)-\frac{7}{t_{g o_{0}}^{3}}\left(v_{x 0}-v_{x f}+a_{x f} t_{g o_{0}}\right)-\frac{1}{t_{g o_{0}}^{2}}\left(a_{x 0}-a_{x f}\right) \\
& c_{3 x}=\frac{6}{t_{g o_{0}}^{5}}\left(x_{0}-x_{f}+v_{x f} t_{g o_{0}}-\frac{a_{x f}}{2} t_{g o_{0}}^{2}\right)+\frac{3}{t_{g o_{0}}^{4}}\left(v_{x 0}-v_{x f}+a_{x f} t_{g o_{0}}\right)+\frac{1}{2 t_{g o_{0}}^{3}}\left(a_{x 0}-a_{x f}\right)
\end{aligned}
$$

Following the same procedure the integration constants for $z$ components $\left(c_{1 z}, c_{2 z}\right.$ and $\left.c_{3 z}\right)$ can be found as

$$
\begin{aligned}
& c_{1 z}=\frac{10}{t_{g o_{0}}^{3}}\left(z_{0}-z_{f}+v_{z f} t_{g o_{0}}-\frac{\left(a_{z f}+g\right)}{2} t_{g o_{0}}^{2}\right)+\frac{4}{t_{g o_{0}}^{2}}\left(v_{z 0}-v_{z f}+\left(a_{z f}+g\right) t_{g o_{0}}\right)+\frac{1}{2 t_{g o_{0}}}\left(a_{z 0}-a_{z f}\right) \\
& c_{2 z}=-\frac{15}{t_{g o_{0}}^{4}}\left(z_{0}-z_{f}+v_{z f} t_{g o_{0}}-\frac{\left(a_{z f}+g\right)}{2} t_{g o_{0}}^{2}\right)-\frac{7}{t_{g o_{0}}^{3}}\left(v_{z 0}-v_{z f}+\left(a_{z f}+g\right) t_{g o_{0}}\right)-\frac{1}{t_{g o_{0}}^{2}}\left(a_{z 0}-a_{z f}\right) \\
& c_{3 z}=\frac{6}{t_{g o_{0}}^{5}}\left(z_{0}-z_{f}+v_{z f} t_{g o_{0}}-F \frac{\left(a_{z f}+g\right)}{2} t_{g o_{0}}^{2}\right)+\frac{3}{t_{g o_{0}}^{4}}\left(v_{z 0}-v_{z f}+\left(a_{z f}+g\right) t_{g o_{0}}\right)+\frac{1}{2 t_{g o_{0}}^{3}}\left(a_{z 0}-a_{z f}\right)
\end{aligned}
$$

The explicit expression of the acceleration commands derived besed on minimum jerk guidance is presented by Eq.(26) and Eq.(27) along with Eq.(30) and Eq.(31).

\section{Simulation Results}

To perform a precise maneuver, the spacecraft trajectory for the terminal descent phase is segmented into two intermediate sub phases, namely the re-targeting phase and vertical descent phase. The re-targeting phase starts from $100 \mathrm{~m}$ altitude and drives the space vehicle and place it just above the re-targeted location of about $20 \mathrm{~m}$ altitude. The segmented spacecraft trajectory at $20 \mathrm{~m}$ altitude provides an additional safety margin to prevent subsurface travel. At the end of re-targeting phase, the guidance algorithm needs to ensure terminal positional accuracy and nullify the horizontal velocity component. Also the spacecraft orientation(look angle) needs to be vertical with respect to the local landing site as shown in Fig.(1). The following numerical parameters, as described in Table(1) has been considered for numerical simulation of the terminal descent phase. The guidance command for re-targeting phases has been designed based on the formulated acceleration commands as given in Eq.(26) and Eq.(27). The coefficients of the acceleration polynomials Eq.(30) and Eq.(31) are computed based the initial and terminal state variables as given in Table.(1). During the vertical descent phase the spacecraft motion is made restricted in $z$ direction only. Hence, $a_{x}$ is considered to be zero. The guidance command $a_{z}$ for the vertical descent phase is obtained using Eq.(27) and Eq.(31). The simulation has been carried out with the spacecraft equation of motion 


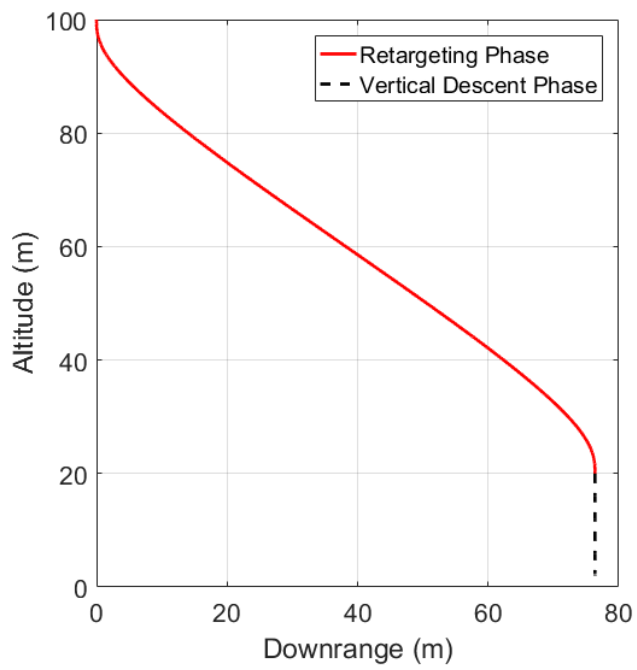

Figure 2. Spacecraft Trajectory

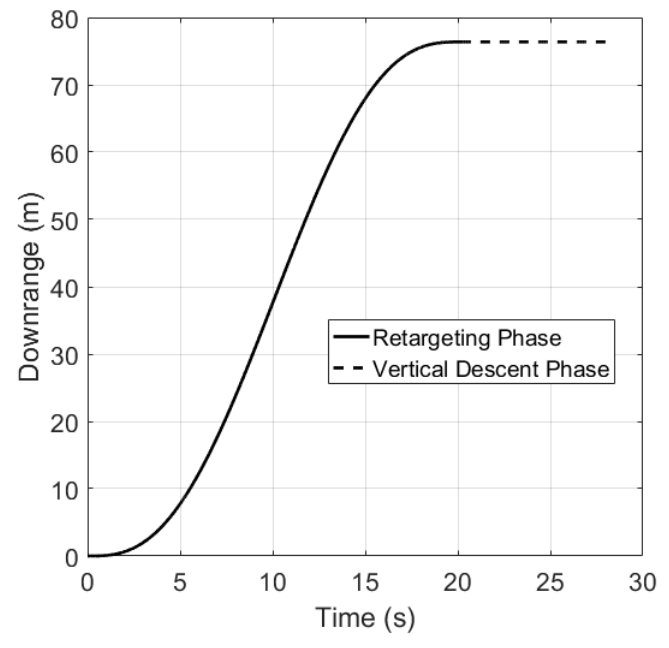

Figure 3. Variation of downrange with time

Table 1. Boundary conditions for Terminal Descent Phases

\begin{tabular}{|c|c|c|c|c|}
\hline Variables & $\begin{array}{c}\text { Re-targeting phase } \\
\text { Initial conditions }\end{array}$ & $\begin{array}{c}\text { Re-targeting phase } \\
\text { Terminal conditions }\end{array}$ & $\begin{array}{c}\text { Vertical descent phase } \\
\text { Initial conditions }\end{array}$ & $\begin{array}{c}\text { Vertical descent phase } \\
\text { Terminal conditions }\end{array}$ \\
\hline$x$ & $0 \mathrm{~m}$ & $76.36 \mathrm{~mm}$ & $76.36 \mathrm{~m}$ & $76.36 \mathrm{~m}$ \\
$z$ & $100 \mathrm{~m}$ & $20 \mathrm{~m}$ & $20 \mathrm{~m}$ & $2 \mathrm{~m}$ \\
\hline$v_{x}$ & $0.1 \mathrm{~m} / \mathrm{s}$ & $0 \mathrm{~m} / \mathrm{s}$ & $0 \mathrm{~m} / \mathrm{s}$ & $0 \mathrm{~m} / \mathrm{s}$ \\
$v_{z}$ & $-2 \mathrm{~m} / \mathrm{s}$ & $-2 \mathrm{~m} / \mathrm{s}$ & $-2 \mathrm{~m} / \mathrm{s}$ & $0 \mathrm{~m} / \mathrm{s}$ \\
\hline$a_{x}$ & $0 \mathrm{~m} / \mathrm{s}^{2}$ & $0 \mathrm{~m} / \mathrm{s}^{2}$ & $0 \mathrm{~m} / \mathrm{s}^{2}$ & $-1.62 \mathrm{~m} / \mathrm{s}^{2}$ \\
$a_{z}$ & $-1.62 \mathrm{~m} / \mathrm{s}^{2}$ & $-1.62 \mathrm{~m} / \mathrm{s}^{2}$ & $-1.62 \mathrm{~m} / \mathrm{s}^{2}$ & \\
\hline
\end{tabular}

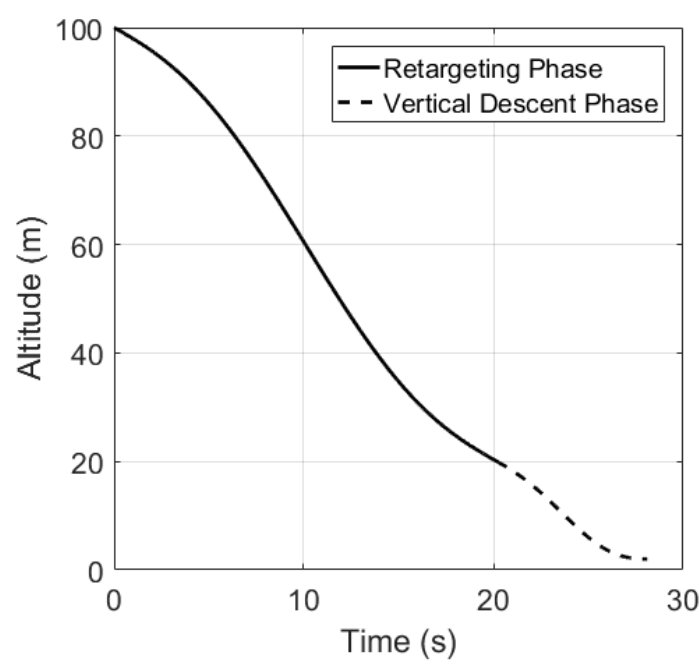

Figure 4. Variation of altitude with time

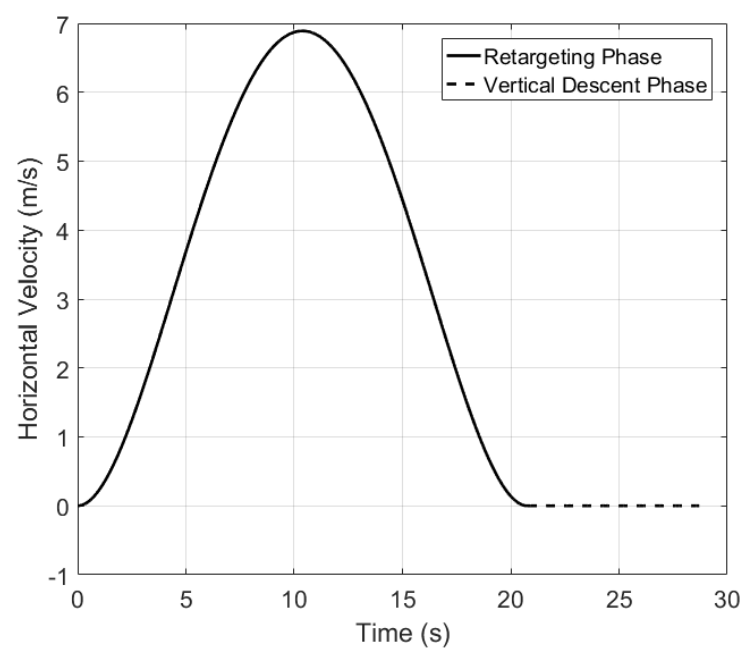

Figure 5. Time history of horizontal velocity

as given in Eq.(1). The gravitational acceleration is considered to be $g=1.62 \mathrm{~m} / \mathrm{s}^{2}$. The curvature of spacecraft trajectory also reduces due to minimum rate of change of acceleration requirement. A trajectory 


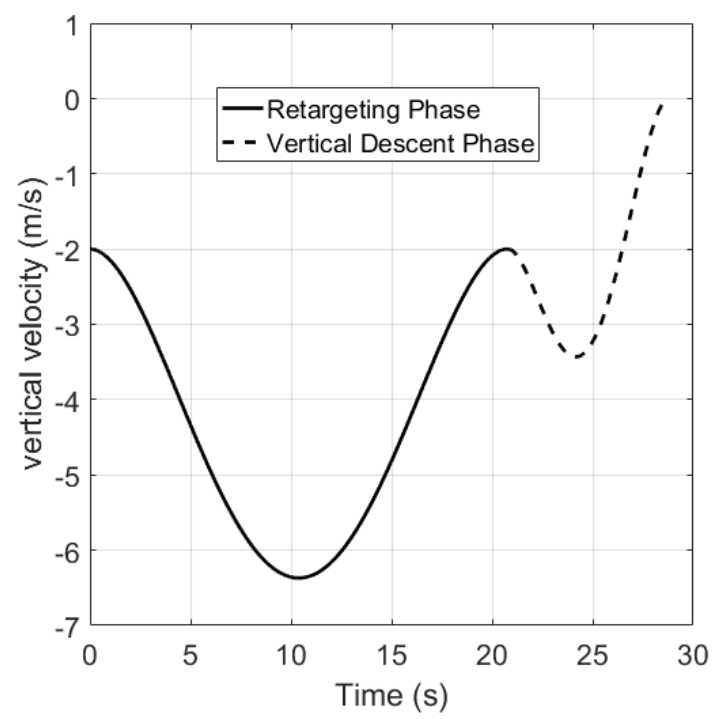

Figure 6. Time history of vertical velocity

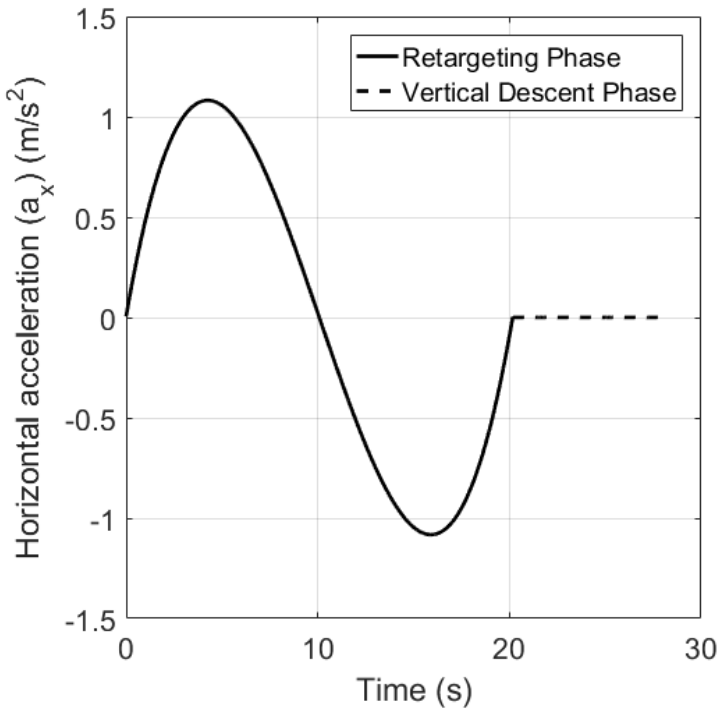

Figure 7. Horizontal acceleration command

with reduced curvature substantially eliminates the possibility of subsurface travel. Simulation results for the spacecraft position, velocity and acceleration are shown in Fig.(3)-Fig.(2). In the plots, solid lines represent the results obtained for the re-targeting phase and dashed lines denote the corresponding profiles for vertical descent phase respectively. The time response of the altitude and downrange profiles are shown in Fig.(3) and Fig.(4).

It is evident from Fig.(3) that at the end of re-targeting phase, spacecraft covers the desired downrange

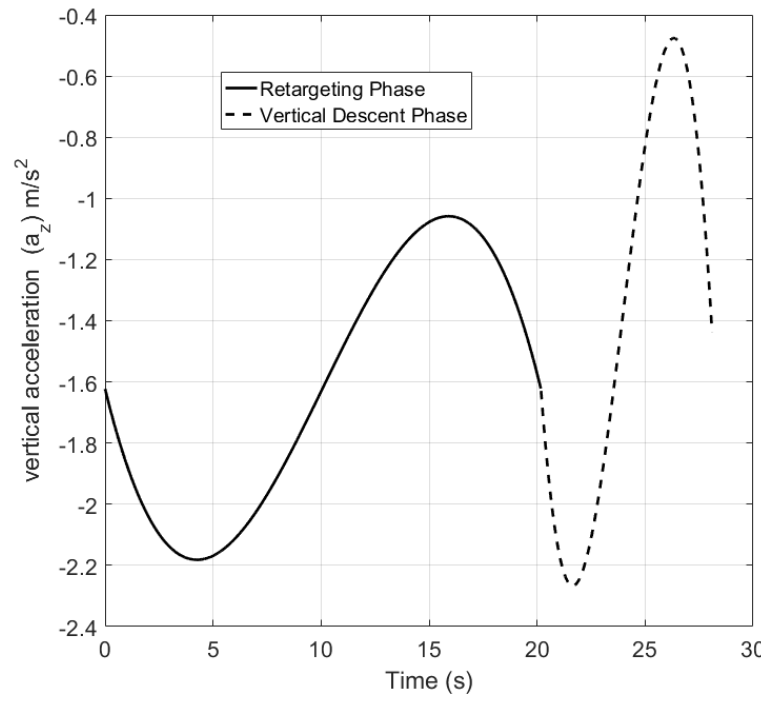

Figure 8. Vertical acceleration command

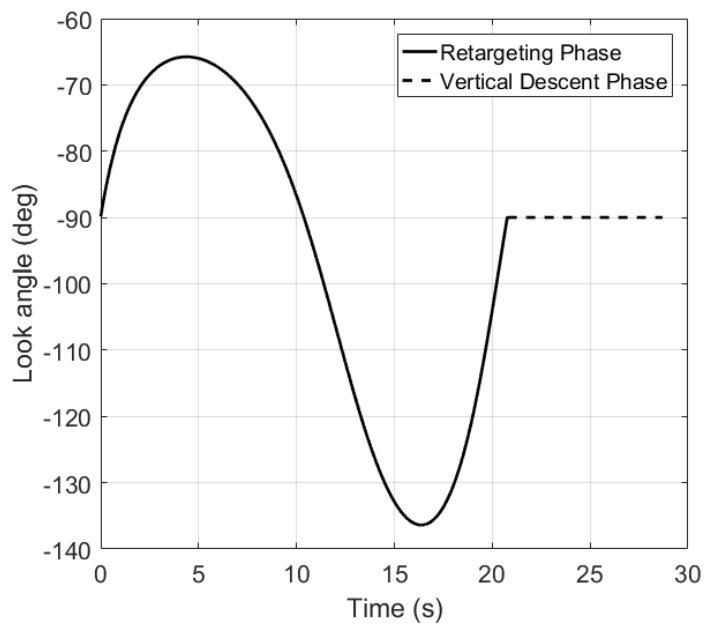

Figure 9. Look angle profile

distance and smoothly placed above the desired landing site at an altitude of $20 \mathrm{~m}$ without any subsurface maneuver as shown in Fig.(2). The result obtained in Fig.(3) also ensures the spacecraft motion is restricted in horizontal direction during vertical descent phase. The simulated spacecraft trajectory shown in Fig.(2) describes a smooth $S$ shaped curve for re-targeting phase followed by the vertical descent(doted line). The velocity profiles are given in Fig.(5) and Fig.(6). During the re targeting phase the horizontal velocity 
builds up to cover the horizontal distance towards the re-targeted landing site. After the re-targeting phase the horizontal component smoothly reaches to zero and stays there. To avoid an additional velocity build up to cover the remaining $20 \mathrm{~m}$ altitude, a residual terminal velocity of $v_{z f}=-2 \mathrm{~m} / \mathrm{s}$ is considered as terminal vertical velocity for the re-targeting phase. This residual velocity $(-2 \mathrm{~m} / \mathrm{s})$ also guides the spacecraft trajectory towards the smooth $S$ shape curve. The minimum jerk based formulation considers the acceleration command as state variable and hence ensure the terminal acceleration constants as shown in Fig.(7) and Fig.(8) which ensures the desired look angle constant(Fig.(9)). Also the the acceleration commands between two intermediate phases are continuous which leads to the smooth spacecraft trajectory.

\section{Conclusion}

Minimum jerk based explicit guidance algorithm for terminal descent phase is presented for the lunar soft landing. Based on the minimum jerk solution, a closed form expression for acceleration command as physical control variable for guidance loop has been derived. The formulation inherently poses the terminal state and control requirements as hard constraints and leads to high terminal accuracy of position, velocity and ensure the vertical orientation of the lunar module. Although, the minimum jerk guidance along with explicit acceleration expression as physical guidance command formulated in this paper is demonstrated for the lunar soft landing mission, the formulation is quite generic and it can address similar class of problems with desired terminal state and control objectives.

\section{Acknowledgements}

The work has been carried out in the DST-FIST supported Advanced Flight Simulation Lab in the Indian Institute of Science and was supported by the research grant from the Indian Space Research Organization (ISRO). The authors would like to thank Shyam Mohan M, Project Associate, Indian Institute of Science, for technical discussions and and motivational support for this work

\section{References}

\footnotetext{
${ }^{1}$ McInnes, C. R., "Path shaping guidance for terminal lunar descent," Acta Astronautica, Vol. 36, No. 7, 1995 , pp. 367-377.

${ }^{2}$ Citron, S. J., Dunin, S., and Meissinger, H. F., "A terminal guidance technique for lunar landing," Aiaa Journal, Vol. 2, No. 3, 1964, pp. 503-509.

${ }^{3}$ Zhang, F. and Duan, G.-R., "Integrated translational and rotational control for the terminal landing phase of a lunar module," Aerospace Science and Technology, Vol. 27, No. 1, 2013, pp. 112-126.

${ }^{4} \mathrm{Xu}, \mathrm{M} ., \mathrm{He}, \mathrm{Y} ., \mathrm{Liang}, \mathrm{Y}$. , and Ding, X., "Terminal guidance strategy for a hybrid thrust-tether lunar landing scheme," Advances in Space Research, Vol. 55, No. 9, 2015, pp. 2280-2292.

${ }^{5}$ DSouza, C., "An optimal guidance law for planetary landing," American Institute of Aeronautics and Astronautics, Vol. 3709, 1997, pp. 1376-1381.

${ }^{6}$ Guo, Y., Hawkins, M., and Wie, B., "Optimal feedback guidance algorithms for planetary landing and asteroid intercept," Proceedings of AAS/AIAA Astrodynamics Specialist Conference, July-August 2011, pp. 569-588.

${ }^{7}$ Ramkiran, B., Preethi, R., Rijesh, M. P., Kumar, G. V. P. B., Philip, N. K., and Natarajan, P., "Analytical optimal guidance algorithm for lunar soft landing with terminal control constraints," Proceedings of Indian Control Conference, Jan 2016, pp. 481-486.

${ }^{8}$ Zhang, B., Tang, S., and Pan, B., "Multi-constrained suboptimal powered descent guidance for lunar pinpoint soft landing," Aerospace Science and Technology, Vol. 48, 2016, pp. 203-213.

${ }^{9}$ Uchiyama, K., Shimada, Y., and Ogawa, K., "Minimum-jerk guidance for lunar lander," Transactions of the Japan Society for Aeronautical and Space Sciences, Vol. 48, No. 159, 2005, pp. 34-39.

${ }^{10}$ Grinfeld, N. and Ben-Asher, J. Z., "Minimal-Jerk Missile Guidance Law," Journal of Guidance, Control, and Dynamics, Vol. 38, No. 8, 2015, pp. 1520-1525.

${ }^{11}$ Mondal, S. and Padhi, R., "Selection of optimal time-to-go in generalized vector explicit guidance," Proceedings of IEEE Conference on Control Applications, Sept 2015, pp. 756-761.
} 